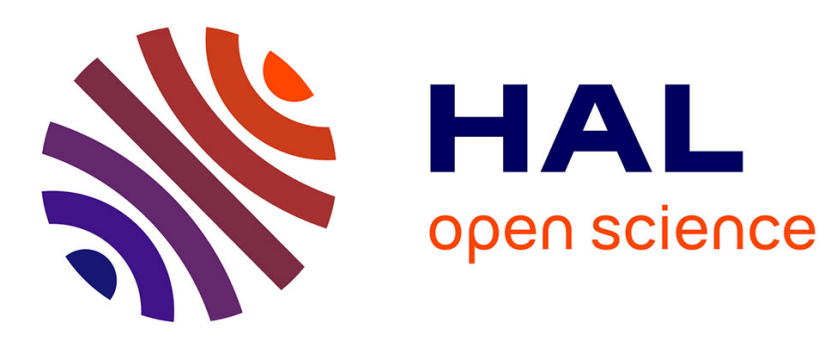

\title{
Les migrantes du care dans l'Europe divisée
}

\author{
Helma Lutz, Ewa Palenga-Mollenbeck
}

\section{To cite this version:}

Helma Lutz, Ewa Palenga-Mollenbeck. Les migrantes du care dans l'Europe divisée: Liens et contradictions dans un espace transnational. Revue des Sciences sociales, 2014, Vers une société du CARE?, 52, pp.18-27. hal-01294985

\section{HAL Id: hal-01294985 \\ https://hal.science/hal-01294985}

Submitted on 30 Mar 2016

HAL is a multi-disciplinary open access archive for the deposit and dissemination of scientific research documents, whether they are published or not. The documents may come from teaching and research institutions in France or abroad, or from public or private research centers.
L'archive ouverte pluridisciplinaire HAL, est destinée au dépôt et à la diffusion de documents scientifiques de niveau recherche, publiés ou non, émanant des établissements d'enseignement et de recherche français ou étrangers, des laboratoires publics ou privés. 
REVUE DES

SGIENGES

SOMMAIRE

VERS UNE SOCIÉTÉ DU CARE? 2014 n52

PRÉSENTATION

p.4 BERNARD WOEHL, PHILPPE HAMMAN \& FREDDY RAPHAËL

p. 8 JUAN MATAS

p.80 CAROLINE GIACOMONI, HÉLEENE HOARAU

.12 DANIEL BERTAUX \& CATHERINE DEL CROIX

DES FLUX MIGRATOIRES DE TRAVAILLEUSES DU CARE

p. 18 HELMA WTZ \& EWA PALENGA-MÓLLENBECK divisée. Liens et transnationa

JUAN MATAS

(n)

D. 42 NATACHA BORGEAUD-GARCIANDA Relations de care, affects ef dominations. Le care à

FRANCE -ALLEMAGNE

ÉTUDES COMPARATIVES DE FORMES DE CARE

D.54 JANINA GLAESER

Assistmilesen methe et politiques de la petite entance : une comparaiso France-Allemagne

p.62 JULLE SENTIS

Apprendre les métiers du care en France et en Allemagne

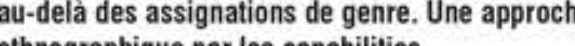

LE CARE EN INSTITUTION

TROIS ÉTUDES DE CAS EN FRANCE

p.72 MOUNRFARHAT

MOUNIR FARHAT personnel et distance professionnelle en unité Alzheimer

P. 80 CAROLINE GIACOMONI, HÉLEENE HOARAU
\& ALAIN MONNEREAU Enjeux et conflits autour du care à travers l'expérience de la délégation à domicile des traitents en concérologie

p. 86 LAURELINE COULOMB Léthique du care malmenée au cours des interactions entre infirmiers et individus sans-abri LUTTES ET RÉFORMES POTENTIELLES

p. 94 URSULA APITZSCH net l'ordre de genre

p. 102 AGNËS BERTHELOT-RAFFARD La discrimination par association : une expression du care dominé

p. 110 CHANTAL NICOLE-DRANCOUR Pour une reconnaissance du care dans des sociétés de pleine activité

p. 118 DANEL BERTAUXX

政 CHANTIERS DE RECHERCHE

p. 132 ÉLOOIE VALENTIN

Emotions, dynamiques citoyennes et espace public. L'expérience du projet social d'une maison de quartier a Dunkerque

p. 142 SUZY GUTH Conflit dans le vignoble : Ammerschwihr et l'AOC Kaefferkopf

LU - À LIRE

p. 154 Recensions

p. 166 Résumés des articles

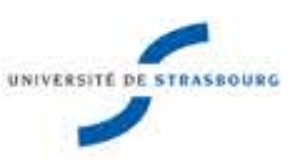

Cirs

Revve bénéficiant du soutien de l'Isstitut
des Sciences Humaines el Sociales du CNRS
SSN 1623-6572

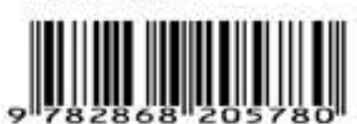

REVUE DES SOIENOES SOBALES

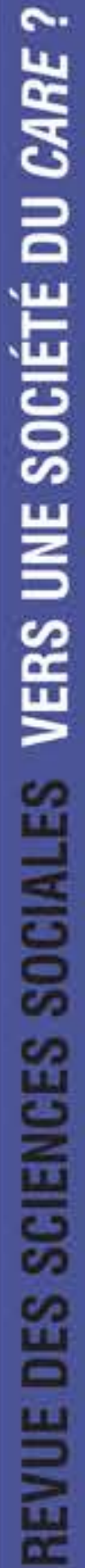

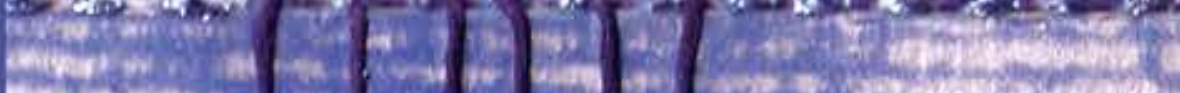

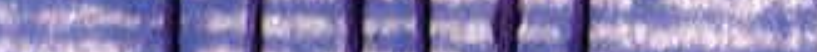

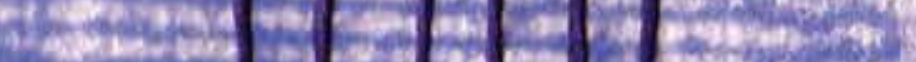
6.

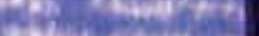
wast:

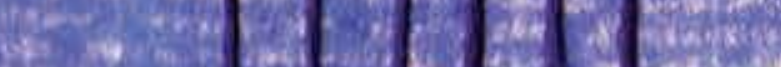

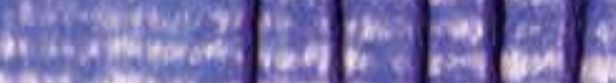

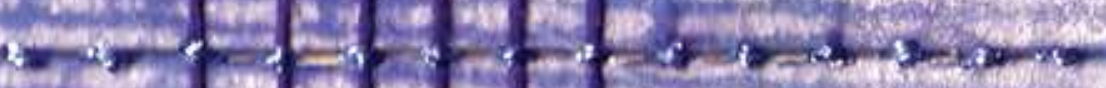
-

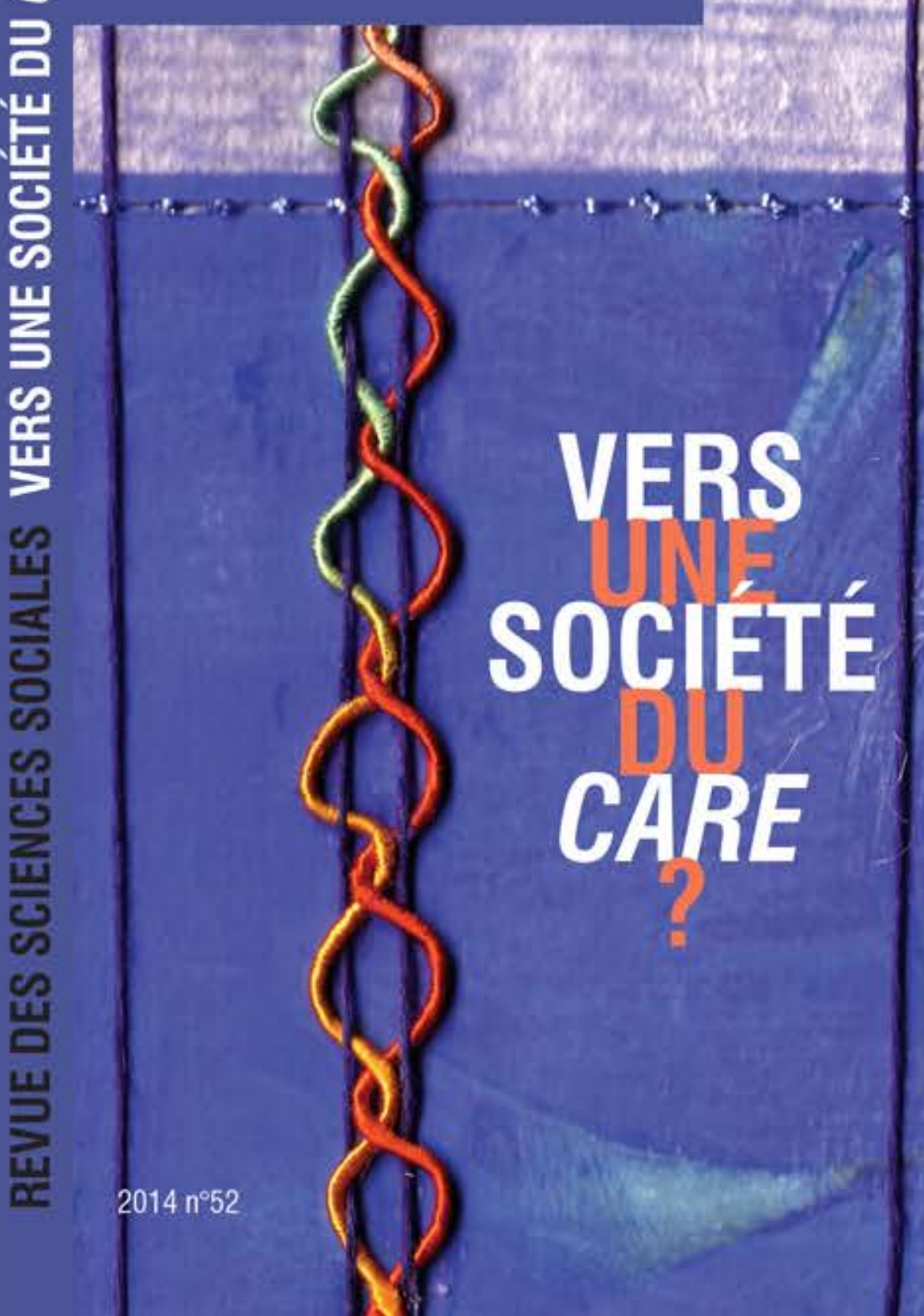


REVUE DES

SAIN

SOGALES

VERS UNE SOCIÉTÉ DU CARE ?

$2014 n^{\circ 5}$ ?

- Directeur scientifique

Freddy Raphaël

- Rédacteur en che

Patrick Ténoudji

- Comité scientifique

Gerges Balandier (EHESS Paris)

(CENRS Toulouse).

(MaymTP Paris) Gionas Paris), Jean Cuisenier

Milano). Jose Carios Gomes da Silva (Portugal).

François Hèran (INED Paris), Claude Javeau (Univ. Libre

de Bruxelles). Nicole Lapierre (EHESS/CNRS Paris).

Marianne Mesnil (Univ. Libre de Bruxelles).

Sonia Montecino (Univ. de Chile), Jean Rèmy (Univ.

Alain Tarrius (Univ. Toulouse-Le Mirail), Alain Touraine

(CEMS Paris)

- Comité de rédaction

Chelle Biannuis-Gasser (Univ. Tours), Maurice Blane Univ. Strasbourg), Nicoletta Diasio (Univ. Strasbourg). Woltong Strasbourg). Antida Gazzola (Univ. Genova). Philippe Hamman (Univ. Strasbourg). Pascal Hintermeyer (Univ. Strasbourg), Leila Jeolas (Uniy Londrina), Reinhard Johler (Univ, Túbingen). Salvatore La Mendola (Univ. Padova), David Le Breton (Univ. Strasbourg/UF), Juan Malas (Univ. Strasbourg. Gabriele Profita (Univ. Palerme), Ilario Rossi (Univ. Lausanne), Patrick Schmoll (CNRS Strasbourg), Roger Some (Uni. Slasbourg), Simona tersign

Collaborateurs éditoriaux

Anny Bloch, Marie-Noële Dents,

Anny Bloch, Marie--Noële Der
- Équipe iconographique sur ce numéro

Vincent Hans, Aline Mathy, Patrick Schmo

- Ce numero a été coordonné par :

Catherine Delcroix, Juan Matas \& Daniel Bertaux

- Maquette

Couverture : Aline Mathy

Presses Universitimires de Stroshour

- Administration

Presses Universitaires de Strasboutro

5 allée du Gal Rouvillois - CS 50008

FR-67083 Strasbourg Cedex

Tél. 0368856265

e-mail : pu-strasbourg@unistra.tr

- Diftusion/distribution

agences d'abonnement :

cid@msh-paris.tr

(nstitutions:

www.lcdpu.fi

18 rue Robert-Schuman

CS 90003

FR-94227 Charenton-le-Pont Ceder

Tel. 0153485630

Revue publiée par la Faculté des sciences sociale

de TUniversité de Strasbourg et le laboratioire

DST 1023.0572 (2)

www.revue-des-sciences-sociales.com
Ál'attention des auteurs

Les articles sont à adresser par document attachẻ à l'attention de Patrick Ténouditi, rédacteur en chêt <patrick.tenoudji@misha.fr>

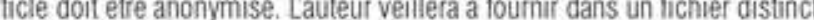

- sestitres ou protession et son appantenance instiutionnelle (université, centre de recherche)

- un résumé d'une dizaine de lignes en français et en anglais.

Le texte comporte au maximum 40000 signes et blancs, notes et bibliographie comprises. II est Myhme par des intertitres courts, avec deux niveaux dintertitres au maximum. Les passages cites de rextes en langues tlangertes sont traduns en lançais. Les nolts, oni

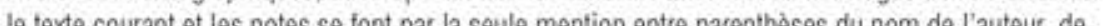
l'année de publication et, le cas échéant, des numéros de pages citées.

Exemples

- ... a ainsi que lindique J. Dupont (2003a) ...

-.... cette question a lairt objel de plusieurs travaux (Oupont 2003a, Durand 2004. 2007) ...

La bibliographie suit les consignes de présentations suivantes :

- pour un ouvage . Oupont J. (2003a), Thre de rouvrage en iltafique, Lieu d'édition, Editeur.

- pour un chapitse douvrage : Durand M. (2004). Titre du chapitre sans guillemets, in Dupont

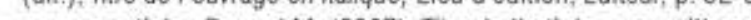

-

Les articles soumis par les auteurs sont examinés chacun par un comité d'au moins deux lecteurs qui rendent des avis séparés. Lexemplaire du texte qui leur est remis ne mentionne pas lidentité de Tauteur, et lauteur de son cote ne peut se laire communiquer T identitie de ses lecteurs : la decision

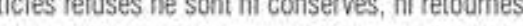

Si des graphiques et illustrations sont nécessaires à l'appui du texte, l'auteur en adresse les originaux ou les fichiers image haute résolution (300dpi) à la rédaction. Hors ce cas de figure, la recherche la rédaction de la revue. 


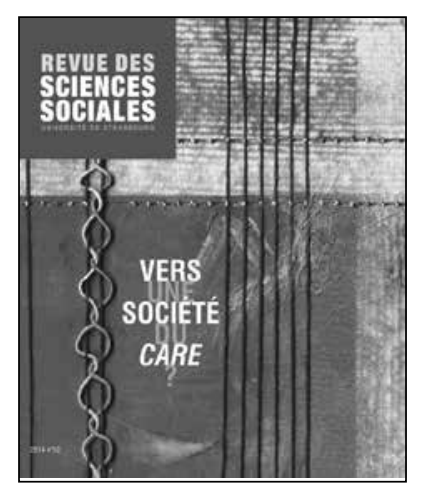

Khadija Seddiki

artiste plasticienne, diplômée de l'Académie royale des beaux-arts de Belgique, de l'Académie des arts et métiers

Constantin Meunier d'Etterbeek, des Écoles supérieures des beaux-arts d'Alger et d'Oran, et de l'Université Denis Diderot-Paris VII. Elle a fondé TISSART, école d'art et atelier de tissage, à Vanves. http://khadijaseddiki.com/
Dans les sociétés du "Nord" de la planète, les besoins de care augmentent alors que les femmes n'acceptent plus guère de s'y consacrer sans contrepartie. Ce déséquilibre engendre des flux importants et différenciés de migrantes venant de «l'Est» ou du «Sud». Qui sont-elles, dans quelles conditions viennent-elles, dans quels cadres, lieux, rapports et conditions concrètes travaillent-elles, quels sont leurs droits?

Ce numéro examine la situation de travailleuses du care: Polonaises en Allemagne, Péruviennes à Buenos Aires ou au Chili; il aborde les contextes institutionnels de ces activités en France, en Allemagne, dans une perspective comparative. Une "société du care» est-elle en train d'émerger?
Couverture

image: Khadija Seddiki composition: Aline Mathy 


\section{PRÉSENTATION}

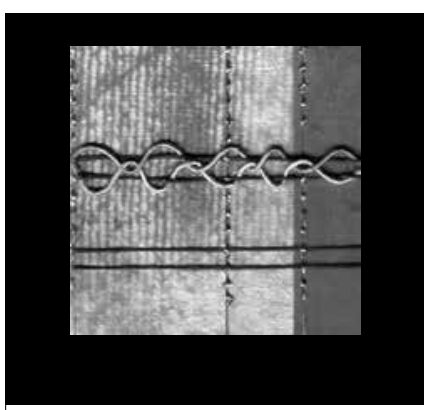

4

BERNARD WOEHL

PHILIPPE HAMMAN \& FREDDY RAPHAËL

Hommage

à François Steudler 8

JUAN MATAS

Introduction

12

DANIEL BERTAUX \& CATHERINE DELCROIX

Présentation
DES FLUX MIGRATOIRES DE TRAVAILLEUSES DU CARE

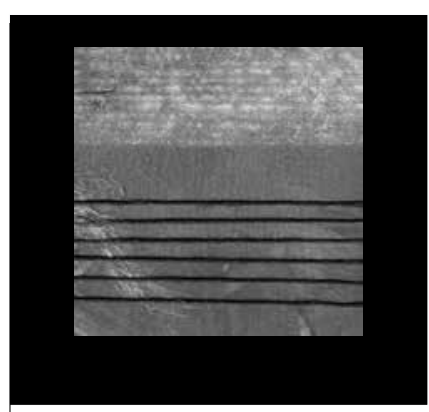

18

HELMA LUTZ \&

EWA PALENGA-

MÖLLENBECK

Les migrantes du care dans

l'Europe divisée. Liens et

contradictions dans un

espace transnational

\section{8}

\section{JUAN MATAS}

Les migrantes péruviennes au Chili et le travail de care

opportunités et risques

d'une situation en plein essor

\section{2}

NATACHA BORGEAUD-

\section{GARCIANDA}

Relations de care, affects et dominations. Le care à demeure à Buenos Aires
FRANGE -ALLEMAGNE : ÉTUDES COMPARATIVES DE FORMES DE CARE

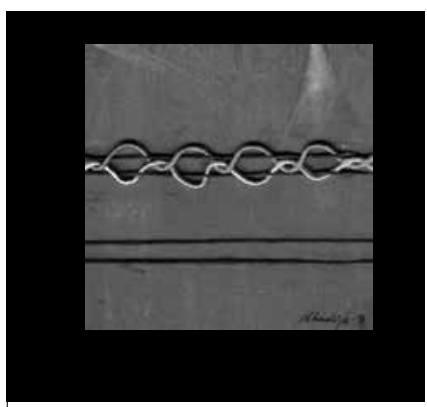

54

\section{JANINA GLAESER}

Assistant(e)s maternel(le)s d'origine étrangère et politiques de la petite enfance: une comparaison France-Allemagne

\section{2}

\section{JULIE SENTIS}

Apprendre les métiers du care en France et en Allemagne au-delà des assignations de genre. Une approche ethnographique par les capabilities
LE GARE EN INSTITUTION : TROIS ÉTUDES DE CAS EN FRANGE

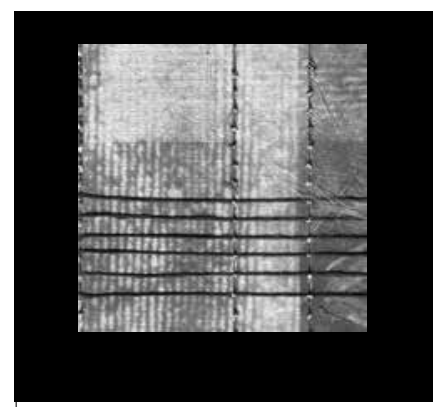

72

\section{MOUNIR FARHAT}

Un dilemme du care:

tension entre engagement personnel et distance professionnelle en unité Alzheimer

80

CAROLINE GIACOMONI, HÉLĖNE HOARAU \& ALAIN MONNEREAU

Enjeux et conflits

autour du care à travers

l'expérience de la délégation à domicile des traitements en cancérologie

86

\section{LAURELINE COULOMB}

L'éthique du care malmenée au cours des interactions entre infirmiers et individus sans-abri 


\section{LUTTES ET RÉFORMES POTENTIELLES}

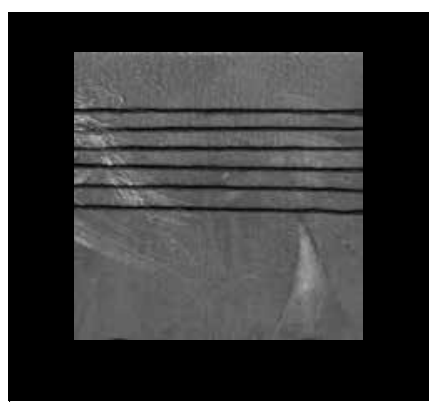

94

URSULA APITZSCH

Le care, la migration

et l'ordre de genre

102

AGNÈS BERTHELOT-RAFFARD

La discrimination

par association:

une expression

du care dominé

110

CHANTAL NICOLE-

\section{DRANCOURT}

Pour une reconnaissance

du care dans des sociétés

de pleine activité

\section{8}

\section{DANIEL BERTAUX}

Le care comme partie

émergée de la production

de la vie

\section{CHANTIERS} DE RECHERCHE

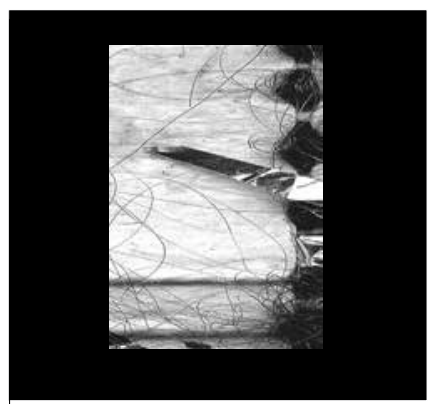

132

ELODIE VALENTIN

Émotions, dynamiques

citoyennes et espace public. L'expérience du projet social

d'une maison de quartier à

Dunkerque

\section{2}

\section{SUZY GUTH}

Conflit dans le vignoble:

Ammerschwihr

et I'AOC Kaefferkopf
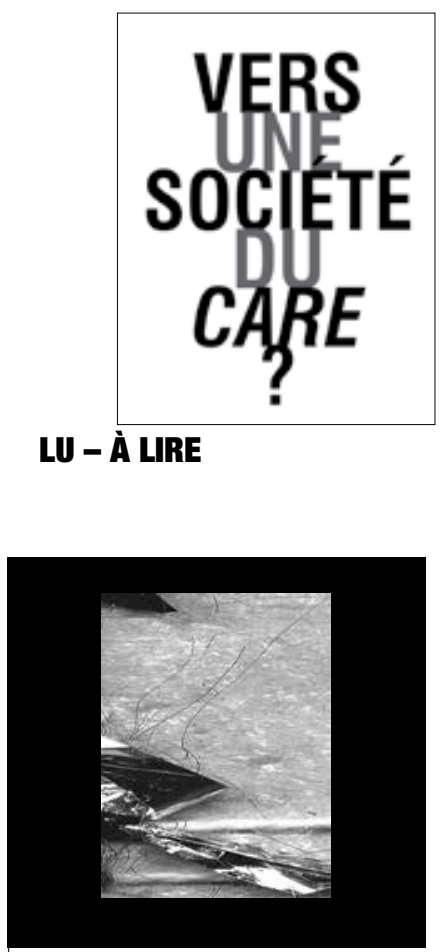

154

RECENSIONS

166

RÉSUMÉS DES ARTICLES 


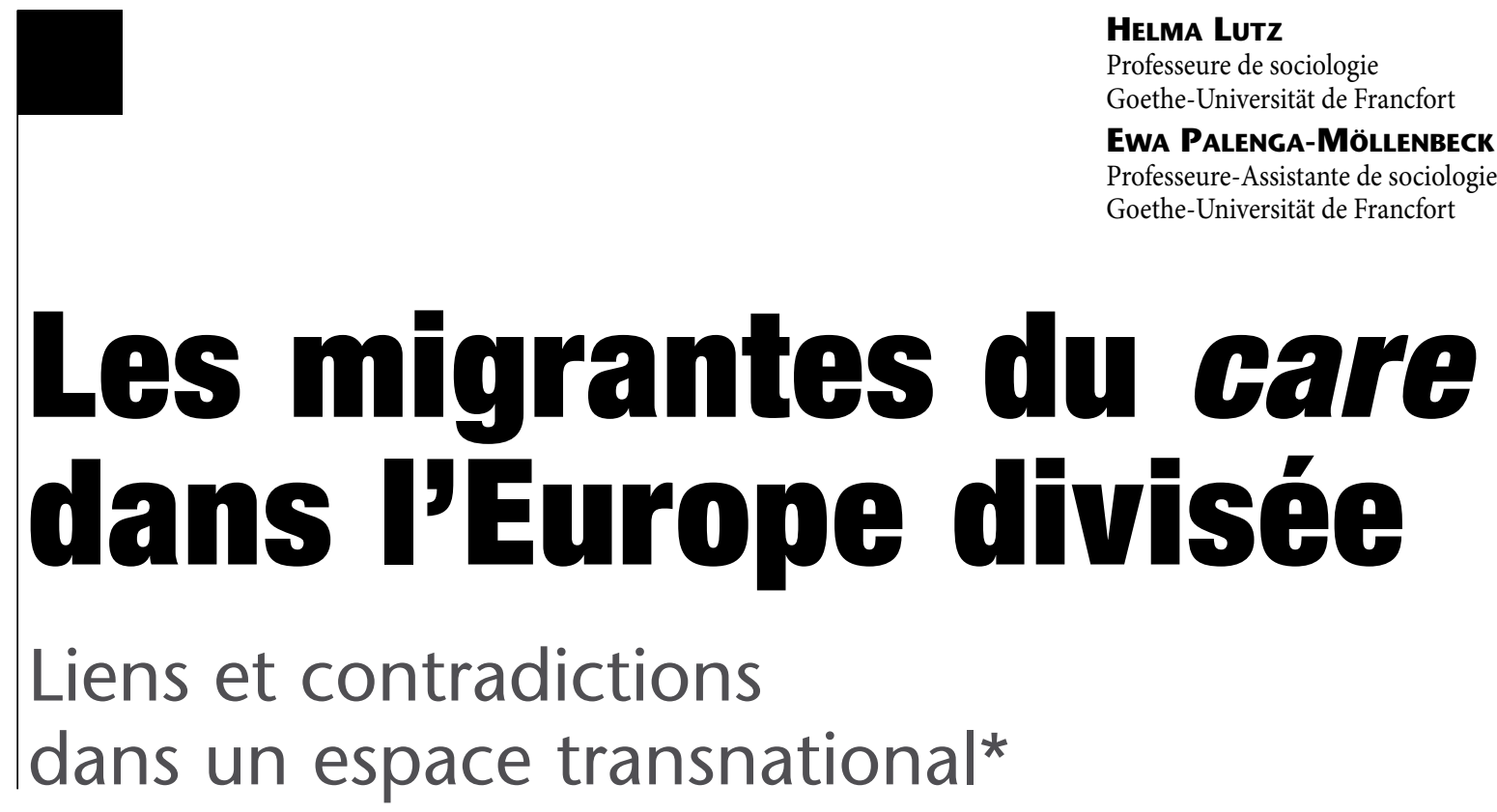

a chute du rideau de fer en Europe remonte désormais à plus de vingt ans. L'Allemagne a été le premier pays à se réunifier avec l'adhésion de l'ancienne RDA à la République fédérale en 1990. La première entrée formelle dans l'Union européenne de pays d'Europe de l'Est (l'Estonie, la Lettonie, la Lituanie, la Pologne, la République Tchèque, la Slovaquie, la Hongrie et la Slovénie) s'est opérée en 2004, suivie d'une seconde vague (la Bulgarie et la Roumanie) en 2007. Cet élargissement s'est poursuivi en 2013 avec l'adhésion de la Croatie. D'autres pays candidats sont la Serbie, la Macédoine et le Monténégro.

Les réglementations juridiques et sociales de l'Union européenne s'appliquent en principe à ces États membres; dans la pratique, cependant, les conditions de vie et d'emploi des pays membres de l'UE sont loin d'être harmonisées.

L'asymétrie entre la dite "vieille» et la «nouvelle» Europe est particulièrement manifeste dans le secteur de la politique du care, qui désigne un aspect de la politique de l'État-providence dont les acteurs principaux sont le trio composé de l'État, de la famille et du marché (Esping-Andersen 1990). La politique du care de l'État-providence se caractérise dans de nombreux pays de "l'ancienne» UE par le fait que l'État n'assure des services dans la prise en charge d'enfants, de personnes âgées et de personnes dépendantes que dans les domaines dans lesquels la famille - ce qui signifie avant tout les femmes ne veut ou ne peut pas assumer cette prise en charge. ${ }^{1}$

L'activité professionnelle croissante des femmes en Europe de l'Ouest et du Sud, une population vieillissante en augmentation et un déficit au niveau des tâches du care qui s'est creusé puisque ces tâches, qui étaient auparavant effectuées gratuitement par les femmes, n'ont été relayées ni par une redistribution entre les sexes, ni par l'État, ont mené à une situation qui a été caractérisée comme "crise du care» (voir par exemple Winker 2011).

Les réactions à cet "état d'urgence» diffèrent fortement d'un pays à l'autre, non seulement celles d'Étatsprovidences différents, mais aussi au sein même des pays concernés. Les membres de la couche moyenne aisée ont davantage les moyens d'acheter des services de care sur le marché que les familles ouvrières (Minijobzentrale 2011). Cette observation s'applique à la plupart des pays industrialisés de l'Ouest; mais on constate en Europe l'émergence d'une nouvelle configuration géopolitique: un clivage qui suit les contours de l'ancien rideau de fer. Il se caractérise par une "perte de care» en Europe de l'Est et par un "gain de care» en Europe de l'Ouest et du Sud où le travail de care est traditionnellement défini comme une tâche familiale. Dans le présent article ${ }^{2}$ nous centrerons l'attention sur l'analyse du phénomène de la chaîne du care dans une Europe divisée, au sein de laquelle la majorité des travailleuses du care sont des femmes migrantes qui ne sont pas originaires - par exemple aux ÉtatsUnis - des pays du Sud, mais d'États européens post-socialistes.

Nous partons de la thèse selon laquelle les migrations du Care de l'est vers l'ouest et le sud constituent de facto une solution au déficit de Care des pays d'accueil. Les États nationaux qui importent des services de soins 
ont recours à deux stratégies: soit ils ouvrent leur marché du travail aux employées migrantes, soit ils tolèrent tacitement qu'un marché de travail de care non déclaré émerge et s'étende. Bien que d'un côté l'Union européenne mette en place des mesures encourageant l'égalité des chances et propage le modèle du travailleur adulte ${ }^{3}$ (Lewis 2004) qui vise autant les hommes que les femmes, ne figurent à l'ordre du jour ni mesures ni même débats sur des dispositifs qui garantiraient une répartition équitable des obligations familiales et professionnelles. De plus l'UE a jusqu'à présent ignoré le fossé socio-économique et les énormes disparités entre les pays pourvoyeurs de care et ceux qui en bénéficient, disparités dont souffrent en premier lieu les membres des familles (les enfants et les personnes âgées) restés au pays.

Nous commencerons cet article par une évaluation critique de la dite "crise du care», engendrée par une forte demande et résolue dans les pays européens de l'Ouest par le travail de care fourni par des migrantes. À partir de l'exemple de l'Allemagne, nous décrirons les réactions face à l'état d'urgence dans la prise en charge de personnes âgées. Après nous être penchées sur l'héritage historique du socialisme d'État, nous nous intéresserons à la question de savoir pourquoi un nombre aussi élevé de femmes issues d'Europe de l'Est participe à cette forme de migration, et nous présenterons les dilemmes auxquels font face ces migrantes transnationales du care. Nous conclurons sur une perspective théorique.

\section{Ladite crise du care}

La législation concernant l'égalité des sexes compte parmi les principaux marqueurs de la politique de I'UE. La première stratégie utilisée pour atteindre cet objectif a consisté dans la promotion du travail salarié des femmes. Entre-temps le modèle du travailleur adulte (Lewis 2004) est devenu la norme centrale du mode de participation à la vie civique. Cette stratégie fait de l'activité professionnelle la priorité par rapport aux tâches de care à domicile. Un engagement entier envers le marché du travail est encouragé, valable pour tous les hommes et toutes les femmes adultes en mesure de travailler. Ce scénario est promu au niveau européen et doit être progressivement intégré aux différents systèmes sociaux en s'inspirant du modèle des pays scandinaves. Ce modèle ignore cependant souvent que la pleine intégration des femmes au marché de l'emploi ne peut être réalisée que si la question de la prise en charge des tâches de care a été réglée. Pour que cette question puisse être résolue, un acteur à qui incombe la responsabilité du care doit être désigné: soit l'État, soit la famille (et au sein des familles, quel sexe), soit le marché.

Les divers États européens ont donné à cette question des réponses différentes. L'UE préconise de s'inspirer du modèle scandinave et de sa politique de conciliation entre vie familiale et professionnelle (voir Eurofund 2006), par une offre complète de services publics de prise en charge d'enfants et de personnes âgées. Mais le principal acteur en la matière, ce n'est pas l'UE, c'est chaque État; et c'est ici que les débats s'engagent sur un terrain glissant. En effet, dans la majorité des Etats membres de l'Union européenne, en particulier dans la "vieille Europe $»^{4}$, la mise en avant de la nécessité du plein engagement des femmes dans une activité professionnelle est en contradiction éclatante avec les codes culturels dominants, ceux des conservateurs qui considèrent le travail de care comme une activité féminine avant tout et une caractéristique de l'identité de la femme, et le travail d'assistance et de soins comme typiquement non-masculin (Plomien 2009). Ainsi, le «nouveau» script des rapports de genre promu par l'UE semble, de toute évidence, devoir entrer en conflit avec «l'ancienne» réalité (voir par exemple Saraceno / Keck 2011).

Dans sa recherche critique sur les réformes politiques introduites par l'UE afin de réaliser le "modèle du travailleur adulte», Mary Daly conclut que si les mesures introduites jusqu'à présent ont affaibli le modèle de foyer à revenu unique masculin, elles n'ont aucunement renforcé une harmonisation des rôles et des styles de vie masculins et féminins (Daly 2011, p. 19). Ainsi les réformes conduisent, selon elle, à un modèle familial à double revenu différencié selon le genre plutôt qu'à une répartition égalitaire du travail salarié et du travail de care, qui était à l'origine prônée par le modèle du travailleur adulte.

En résumé, on peut voir dans les résultats de recherche les plus récents que le problème de la répartition asymétrique genrée du travail de care dans les foyers continue d'être crucial dans de nombreux pays, et qu'il n'est "résolu» que par les pays qui peuvent faire appel à la voie du marché (informel). Avec la marchandisation du travail domestique et du travail de soins, les questions touchant à une répartition égale entre les sexes sont perdues de vue; ceci vaut autant pour les foyers et l'espace privé que pour les débats publics sur le déficit du care.

\section{La crise du care et la commercialisation du travail domestique et d'assistance en Allemagne}

L'une des stratégies pour combler le déficit de care consiste à transférer à d'autres les tâches telles que le nettoyage, la cuisine, la lessive, le jardinage, l'encadrement et la garde d'enfants, et les soins aux personnes âgées. Ce processus de transfert se répand en Allemagne comme dans toute l'Europe (Lutz 2008; Isaksen 2010). Selon une étude récente, en 2009 4,5 millions des ménages allemands (environ $11 \%$ de l'ensemble des foyers en Allemagne) ont acheté "des services destinés aux ménages ", $95 \%$ d'entre eux de manière non déclarée (IWK 2009, p. 3). En 2003, le gouvernement allemand avait introduit et promu un programme de soutien étatique, une procédure de remise de chèques aux ménages, en principe pour soulager financièrement ceux qui employaient des aides à domicile mais surtout pour faire sortir le travail domestique, qui continuait d'être effectué 
par des migrantes non déclarées, de sa 'zone grise'. Depuis, le nombre des employées de ménage officiellement employées augmente chaque année; cependant seule une petite proportion d'entre elles dispose d'un contrat (Minijob-Zentrale 2011, p. 3).

En Allemagne la politique actuelle du care (l'encadrement et la garde d'enfants, et les soins aux personnes âgées) continue de reposer avant tout sur le principe du familialisme (Backes et al. 2008, p. 20). Combiné à la prémisse du 'modèle du travailleur adulte' cela amène à un déficit de care considérable. Les ménages aisés peuvent certes le compenser en recrutant des employées (migrantes) à domicile.

En ce qui concerne la prise en charge d'enfants, l'Allemagne, qui est historiquement marquée par le modèle de l'homme soutien du ménage, a mis en place de nombreuses réformes concernant les politiques de la famille depuis les années 1990, notamment en ce qui concerne l'égalité des sexes sur le marché du travail, le développement de l'infrastructure de garde d'enfants, et l'introduction du congé parental (Böllert/ Oelkers 2010). Cependant, en 2010 encore, seuls 20,4\% des moins de 3 ans étaient pris en charge dans des crèches (BMFSFJ 2010, p. 29); et même depuis l'introduction de l'obligation légale de prise en charge, des places en crèches et en maternelles continuent de manquer dans les secteurs de la petite enfance et de l'enfance. Le travail d'encadrement et de garde continue de se faire principalement au sein des familles, qui ont alors recours aux services des grandsparents ou de jeunes migrant-e-s au pair et de babysitteurs-ses (Hess 2005). Quant aux soins aux personnes âgées, la migration sans papiers du care correspond à une part importante du quotidien de l'État-providence, tendance que nous avons décrite comme de la "complicité étatique " (Lutz / PalengaMöllenbeck 2010). $67 \%$ des personnes dépendantes utilisent les prestations qu'elles obtiennent de l'État pour mettre en place des arrangements de soins dans l'espace privé, des services rendus la plupart du temps par des femmes ou par des membres des familles secondés par des prestataires de soins professionnels (Statistisches Bundesamt 2013).

Le faible soutien financier destiné aux services de soins proposés par des prestataires commerciaux, mais aussi les transferts de services directs non contrôlés au sein des familles constituent les raisons principales d'une demande et d'un emploi croissants d'employées migrantes vivant au sein des foyers; un phénomène largement ignoré par le gouvernement. L'écart entre la demande persistante et une politique migratoire restrictive qui définit le travail de care comme une tâche non qualifiée a mené à l'émergence d'un important marché informel de soins (Lutz/Palenga-Möllenbeck 2010).

Nous avons analysé le discours de la presse ${ }^{5}$ sur la thématique «travail de care et migration " à travers l'étude de 279 articles parus entre 1997 et 2008. On y constate une contradiction entre la politique de soins de l'État et l'«état d'urgence du care» dont parle la presse. Dans la FAZ et dans la Bild-Zeitung en particulier, l'emploi de migrantes d'Europe de l'Est est considéré comme une solution non seulement pragmatique (le quotidien Bild Zeitung) mais aussi judicieuse sur les plans politique et économique (le quotidien $F A Z$ ) des problèmes allemands dans le secteur des soins. Dans le quotidien $S Z$ plus critique, un expert évoque en revanche la situation de la façon suivante: «Le système est illégal (...) mais il fonctionne»; et le journaliste conclut: «S’il n'y avait pas les présumées Hongroises et Polonaises, Tchèques et Roumaines qui travaillent de façon illégale, les soins à domicile s'effondreraient. C'est donc toléré, plus ou moins en silence (...)» (Kastner 2008, p. 41).

Une étude réalisée en 2009 estime le nombre des migrant-e-s en situation irrégulière venant d'Europe de l'Est et travaillant dans le secteur des soins aux personnes âgées à 100000 personnes, et le potentiel à 145000 (Neuhaus et al. 2009, p. 9). Et dans de nombreux autres pays européens la situation est peu différente en ce qui concerne les soins aux personnes âgées. En particulier les États d'Europe du Sud, dans lesquels les déficits dans l'assistance et les soins ont particulièrement augmenté (du moins jusqu'à la crise financière) en raison d'une montée de l'activité professionnelle des femmes sur le fond d'un modèle familialiste du care, importent des migrantes du care provenant d'Europe de l'Est. En Italie, on estime le nombre de migrantes originaires de Roumanie, d'Ukraine, de Moldavie et d'Albanie à un million de personnes (ISTAT 2011).

$\mathrm{Au}$ total, on peut donc distinguer deux tendances:

D'une part, la normalisation croissante de la marchandisation du travail de care et simultanément le recours à un marché qui se situe en grande partie en dehors du Code du travail. D'autre part, une tendance persistante à ignorer ce phénomène, qui se traduit par la rareté des données existantes. L'Organisation Internationale du Travail (ILO) signale régulièrement dans ses publications que les données dans le secteur du travail de care et du travail domestique sont mauvaises; alors que la somme des travailleurs-ses domestiques inscrit-e-s sur le plan mondial compte 53 millions de personnes, les estimations partent plutôt de 100 millions (ILO 2013). Cela est également valable sur le plan européen: ni les pays émetteurs de main-d'œuvre, qui caractérisent les employées migrantes du care de "temporairement absentes " et ne les incluent donc pas dans leurs taux d'émigration, ni les pays bénéficiaires de main-d'œuvre, dont les politiques migratoires ne favorisent que le recrutement de personnes dites hautement qualifiées et qui n'ont pas intérêt à abandonner l'illusion d'une "politique d'immigration contrôlée», n’ont intérêt à disposer de données précises.

Nous nous sommes posées la question de savoir ce qui pousse tant de femmes (hautement qualifiées, voir Lutz 2008) d'Europe de l'Est à accepter de s'engager dans cette activité et dans les problèmes sociaux afférents. Un regard sur l'histoire du socialisme aide à répondre à cette question. 


\section{Héritage historique et égalité des sexes dans le (post)socialisme.}

Dans les pays dits de socialisme d'État, les femmes étaient appelées à compléter le revenu de leur ménage. $\mathrm{Au}$ sein de ce système politique, la participation des femmes au marché du travail était la règle et non l'exception. Le socialisme d'État combattait le modèle de la "femme au foyer" en imposant un modèle uniforme «d'obligation d'emploi pour tous les adultes ", modèle que les mouvements féministes dans les pays de l'Ouest ont longtemps qualifié de progressiste (Menschik/Leopold 1974). Depuis la parution en 1884 de l'ouvrage pionnier de Friedrich Engels L'origine de la famille, de la propriété privée et de l'État, dans lequel l'auteur expliquait que la libération de la femme ne serait pas réalisée tant qu'elle resterait exclue du travail productif et qu'elle serait réduite au travail domestique dans l'espace privé (Engels [1884] 1974, p. 181), la branche (socialiste) du mouvement féministe dans toute l'Europe a débattu et revendiqué le droit des femmes à l'accès au travail rémunéré. Les utopistes, les féministes et les socialistes tel-le-s que Rosa Luxemburg, Clara Zetkin et August Bebel percevaient l'emploi des femmes à temps plein et leur libération des obligations de tâches domestiques et de soins comme une clé pour atteindre l'égalité des genres, qui résulterait (automatiquement) du passage du système capitaliste au système socialiste. Dans le socialisme d'État, l'emploi féminin fut régulé à l'aide d'une infrastructure complète (des crèches, des maternelles, des écoles à plein temps, des cantines, des maisons de retraite, etc.) et par des restrictions financières (des revenus plus bas pour des ménages à un seul revenu). Dans la plupart des pays socialistes, plus de $50 \%$ des femmes salariées travaillaient à temps plein; ainsi, en Pologne, par exemple, presque $70 \%$ des femmes mariées étaient salariées au début des années 1980 (Sikorska 2009, p. 92). Ce chiffre était même dépassé dans de nombreux autres pays d'Europe de l'Est. Cependant, la participation élevée des femmes au marché du travail n'a pas forcément conduit à une répartition égale des tâches ménagères et du travail d'assistance et de soins entre les sexes; la question de la conciliation entre les deux resta au contraire un "problème des femmes". Paradoxalement la répartition genrée du travail s'est ainsi conformée à un modèle traditionnel dans le sens où non seulement les femmes salariées, mais aussi leurs mères ou leurs bellesmères retraitées se chargeaient des tâches d'assistance et de soins (Saxonberg / Szelewa 2007, p. 356); car malgré les vastes aménagements réalisés, l'infrastructure de garde et de soins existante était souvent insuffisante (Giza-Poleszczuk 2007, p. 304).

Après 1989, et au gré des réformes néolibérales, l'État se retira des tâches qu'il avait exercées jusque-là et la responsabilité d'assistance fut entièrement confiée aux familles (plus précisément aux membres féminins des familles). Par exemple l'Office Statistique de l'Ukraine a recensé un recul dramatique du nombre d'écoles maternelles, qui passèrent de 2300 en 1991 à 1100 en 2010 (Libanova et al. 2011). Dans tous les pays du bloc postsocialiste on constate une tendance générale à la refamilialisation du travail du care. En raison de suppressions massives de services aux familles sur le plan politique, ainsi que la perte rapide d'emplois dans les secteurs de travail majoritairement féminins (Kałwa 2007, p. 208; van Klaveren et al. 2010), de nombreuses femmes se sont vues contraintes de quitter le marché du travail. Cela correspondait à un renouveau de l'idéologie conservatrice de la «nouvelle maternité » (Szelewa / Polakowski 2008, p. 117). En même temps, on peut relever dans ce processus de transformation un changement paradoxal concernant les rapports intrafamiliaux et de genre: tandis que le modèle traditionnel trouve un soutien sur le plan idéologique et politique, le modèle du foyer à double revenu est devenu en revanche plus que jamais nécessaire à la survie financière des ménages (Giza-Poleszczuk 2007, p. 309).

\author{
De nouveaux schémas \\ migratoires \\ en provenance de \\ et entre les pays \\ d'Europe de l'Est
}

Malgré la fermeture géopolitique d'avant 1989, qui ne prévoyait pas de droit au retour pour les personnes ayant quitté le territoire (les 'réfugiés'), une migration de travail temporaire, saisonnière et majoritairement masculine au sein du bloc socialiste était largement répandue. Au cours de la libéralisation des contrôles des frontières après 1989 , ce modèle se transforma de façon considérable: en raison de la situation économique, la migration transnationale est devenue peu à peu une stratégie d'emploi importante pour une partie (jusqu'à un tiers) de la population non seulement masculine mais surtout féminine.

La migration se dirige de façon générale en direction de la "vieille» Europe, c'est pourquoi dans les pays émetteurs de main-d'œuvre elle est aussi appelée "Euro-migration». La migration de l'Ukraine vers la Pologne, d'un pays non-membre de l'UE vers un pays membre de l'UE, est encouragée par la proximité géographique et les revenus plus élevés des centres urbains polonais, ainsi que par la demande croissante, par des familles de la classe moyenne polonaise, d'employées dans le secteur du care. La majorité de la migration ukrainienne du care se dirige cependant vers des pays de l'Europe du Sud: l'Italie, l'Espagne, le Portugal, la Grèce.

La migration féminine, qui est par son ampleur un phénomène historiquement nouveau dans toute l'Europe de l'Est, a été instaurée par les actrices sous forme de migration pendulaire du travail: les familles restent dans le pays d'origine, et les femmes rentrent régulièrement chez elles selon un cycle de 6 à 12 semaines. Cette forme de mobilité place aujourd'hui les sociétés des États post-socialistes face à un profond dilemme idéologique. D'une part les traditionalistes qui stigmatisent la migration des mères sont devenus, avec le soutien des Églises 
catholique et orthodoxe et des nationalistes radicaux, les nouveaux porteparoles des discours dominants; ils exigent une intervention de l'État en faveur des enfants (voir plus bas), et visent ainsi une restriction de la mobilité des femmes, mais ne réfléchissent aucunement à une nouvelle conception du rôle des pères restés au pays. D'autre part, les devises envoyées par les migrantes revêtent une grande importance pour le budget de l'État de chaque pays d'émigration. Mais de nombreuses familles se voient-elles aussi très fortement contraintes de faire face à une situation financière critique et de compenser en même temps les réductions des dépenses publiques affectées aux systèmes d'éducation et de formation ainsi qu'aux soins et de santé. De façon très paradoxale, les modèles traditionnels d'assistance sont ainsi davantage remis en cause que durant des décennies de socialisme d'État. Pour les femmes migrantes, dont «l'héritage socialiste " consiste dans une bonne formation et la normalité d'une activité professionnelle, le plus grand problème de cette forme de migration ne réside aucunement dans la conciliation entre emploi et famille, mais davantage dans les conditions de sa réalisation: de longues absences physiques loin de leur famille, la perte de droits civiques et sociaux, en particulier en cas de travail non déclaré, et l'absence d'encadrement légal et de contrôle des conditions de travail. Nous illustrerons cela à l'aide de plusieurs exemples tirés de nos études de cas. ${ }^{6}$

\section{Dilemmes et contradictions: la conci- liation entre travail d'assistance et de soins et activité professionnelle dans un espace transnational}

Toutes les migrantes du care que nous avons interviewées, en particulier les mères, rencontrent des difficultés à concilier leurs obligations de mères avec leur emploi dans un foyer à l'étranger. Souvent, elles se sentent tiraillées entre ces deux espaces de responsabilités. Halina, une veuve polonaise âgée de 45 ans qui poursuit une activité professionnelle depuis sa jeunesse, n'est jamais restée à la mai- son plus de trois mois, même après la naissance de chacun de ses trois enfants. Dans l'extrait d'entretien suivant elle évoque les différences entre son activité professionnelle en Pologne et sa migration transnationale actuelle vers l'Allemagne.

"On rentre à la maison, on a été absent un certain temps, c'est chaque fois une réorganisation totale; on aimerait faire un tas de choses et il y a tant à faire, vous savez bien comment cest. Et je dois déjà repartir avant d'avoir tout réglé. Quand jy pense, quand je travaillais encore ici [en Pologne], à l'époque les enfants devaient aller à la maternelle le matin et je devais être au travail à 7 heures; je rentrais à 15 heures, jallais chercher les enfants - oui, cétait un rythme quotidien normal. Et maintenant, chaque fois que je suis ici, enfin à la maison, je veux aller rendre visite à mon père, à ma famille et le temps passe tellement vite, nous navons pas assez de temps les uns pour les autres et voilà que je dois déjà repartir. - Le pire dans tout ça, c'est que je ne suis plus dans ce rythme: être à la maison, l'emploi, le travail ménager, aller à l'église le dimanche, etc."

Pour Halina, une vie sans activité professionnelle n'est pas concevable; et elle insiste à plusieurs reprises sur le fait qu'elle aime travailler. Elle souffre de la séparation d'avec ses enfants mais, en tant que veuve subvenant seule aux besoins de la famille, elle gagne beaucoup plus d'argent en Allemagne.

L'histoire d'Halina est représentative des femmes d'un certain âge ainsi que des femmes plus âgées (voir Solari 2010 , p. 226). Ayant grandi dans la tradition du socialisme d'État, elles pouvaient (et devaient) avoir recours à des institutions publiques de prise en charge afin de pouvoir concilier leurs obligations domestiques et de soins. Ce qui pose problème à Halina, qui vit dans les foyers privés des personnes âgées pour lesquelles elle travaille en Allemagne, c'est qu'elle n'arrive plus à remplir son idéal de "bonne mère " étant donné que celui-ci exige une proximité physique avec ses enfants. Une transformation des idéaux de la figure de la (bonne) mère ne semble suivre ces changements de conditions de vie qu'avec de grands retards - si elle les suit tout court.
De nombreuses jeunes femmes en revanche ont un plan très clair concernant leur avenir, ou du moins présentent leur décision de partir travailler à l'étranger en tant que tel. Ainsi Ala, une jeune femme de 28 ans mère d'un enfant de 5 ans, dont le mari a monté son entreprise artisanale en Pologne. Malgré une formation universitaire elle n'a pas trouvé d'emploi en Pologne; c'est ainsi qu'elle est partie en Allemagne, non pas pour compenser un revenu familial insuffisant, mais pour réaliser plus vite le rêve d'une accession à la propriété privée sous la forme d'une maison individuelle; celle dans laquelle l'entretien finit par se faire:

"Lorsque je suis partie pour la première fois pour aller travailler, je voulais travailler de façon légale, javais un projet pour les deux années à venir. Ces deux années se sont terminées en septembre dernier. J'ai réalisé mon projet, car javais dit de façon très claire: 'Okay, je pars, mais alors nous devrons construire une maison avec cet argent."

Ala, comme d'autres mères, a beaucoup souffert de la séparation d'avec son enfant. Tout comme Halina et de nombreuses autres femmes, elle a tenté d'arracher la permission à son employeur d'amener son enfant avec elle en Allemagne. Mais cette autorisation lui a été refusée, car les employeurs craignaient que la présence de l'enfant ne perturbe le déroulement du travail (les femmes effectuaient un travail de soins 24 heures sur 24) et complique la question du logement. Mais tandis qu'Halina rencontrait de grandes difficultés à trouver quelqu'un qui s'occupe de ses enfants, en particulier de sa plus jeune fille, la mère et la belle-mère d'Ala se relayaient pour s'occuper de son fils. Il était prévu qu'à son retour elle reprenne ces tâches. On peut donc constater, dans le cas d'Ala comme dans d'autres, que ce sont encore une fois les femmes de la famille qui se retrouvent responsables de la prise en charge des enfants.

L'interview avec Wojtek (52 ans), dont l'épouse travaille en Allemagne, montre que les migrantes reçoivent au moins un soutien moral de leur famille, et la manière dont celui-ci s'opère. Avant son départ pour l'Alle- 
magne, Janina travaillait comme chef de service dans une chaîne polonaise de supermarchés; le couple a une fille de deux ans et demi:

"J'ai dit à ma femme: je te connais, toi et ton travail! Même si tu étais dans ce pays [à savoir la Pologne], ce ne serait pas différent. Tu travaillerais 15 ou 16 heures par jour, même les samedis et dimanches, et tu verrais aussi peu ton enfant, et tu travaillerais pour moins d'argent et dans des conditions de travail pires. Laisse tomber! Tu peux refaire le calcul toi-même, si tu veux.»

Cet extrait d'entretien confirme que dans les pays post-socialistes l'emploi des femmes à l'extérieur du foyer est considéré comme une contribution évidente au revenu de la famille, et que la considération du 'travail' comme partie intégrante d'une biographie sert à «normaliser» un mode de vie transnational. Durant le reste de l'entretien, Wojtek met en avant, dans la suite logique de ces propos, les conditions de travail de son épouse en Allemagne et décrit le comportement de ses différents employeurs et leurs répercussions (négatives) sur le moral de cette dernière. Il évoque également la manière dont il l'a conseillée sur comment réagir à ces situations.

L'extrait d'entretien le plus intéressant concernant cette "normalisation » de la migration des mères provient de Dawid, un garçon polonais âgé de 12 ans, qui, interrogé sur qui va promener le chien, répond que cette tâche lui incombe à lui-même :

"Oui, car papa est au travail et maman - aussi. La plupart du temps, je suis seul à la maison."

Dawid voit le point commun entre ses parents dans le fait que tous deux travaillent, même si la distance jusqu'au lieu d'emploi de sa mère est de 800 kilomètres. Mais la courte pause avant le "aussi» indique qu'il ne lui est pas tout à fait facile de le reconnaître...

On peut retenir ici que la «normalisation» de l'absence (par intermittence) de la mère est perçue par toutes les personnes concernées (époux, grands-mères, enfants) comme faisant partie d'un projet commun; et qu'elle contribue de façon importante à élaborer des stratégies permettant de surmonter au quotidien la situation migratoire. Cela soulage les mères, mais leur sentiment de culpabilité demeure.

Un autre aspect s'y ajoute, qui vient contrecarrer le travail de normalisation réalisé dans ces familles: dans de nombreux pays d'émigration d'Europe de l'Est, les discours publics sur les migrations sont de plus en plus dominés par une indignation moralisante au sujet des enfants «laissés seuls», les «Euro-orphelins» (pour la Pologne et l'Ukraine voir Lutz / Palenga-Möllenbeck 2011; pour la Roumanie voir Rerrich 2012). Le terme d'Euro-orphelin', qui qualifie ainsi les enfants des migrantes restés dans leur pays d'origine de quasi-orphelins et qui considère l'absence des parents comme contrevenant aux droits des enfants, provient de la presse polonaise. En Roumanie, on parle «d'enfants délaissés»; en Ukraine, "d'orphelins sociaux»; et en Moldavie, "d'orphelins blancs ». Employés dans les médias depuis 2008, ces termes se sont rapidement transformés en slogans que l'on retrouve dans les articles de presse, sur Internet (en particulier sur Youtube), mais aussi dans des articles scientifiques (par exemple Mikuła 2008 , p. 3); les médias suggèrent qu'il s'agit là d'un phénomène de masse. L'absence physique des parents est décrite comme un phénomène scandaleux, mais les exemples rapportés concernent le plus souvent la "mère absente» (voir Lutz / Palenga-Möllenbeck 2011). Or, comme le souligne le sociologue polonais Walczak (2008), les enfants des migrant-e-s de travail ne sont aucunement des orphelins au sens juridique du terme tant que le parent qui n'a pas émigré ou leur grand-mère s'occupe d'eux. Ce constat vaut pour tous les pays pourvoyeurs de main-d'œuvre. Cependant les protagonistes de ce débat partagent l'idée que les enfants qui ne grandissent qu'avec un seul de leurs parents se comportent comme des orphelins (Dziennik.pl, 27/3/2008).

Pour l'analyse détaillée du débat sur les "orphelins» dans le contexte de la transformation post-socialiste nous devons renvoyer à notre ouvrage à paraître (Lutz 2015); ce qui nous importe ici, c'est le constat que les mères que nous avons interrogées se réfèrent couramment à ces discours qui les contraignent à légitimer davantage leur migration, tout en faisant passer au second plan le fait que leur activité professionnelle et les devises qu'elles génèrent contribuent à compenser le désinvestissement de l'État en matière d'infrastructures publiques dans les secteurs de l'éducation, de la santé et des soins. De plus, non seulement les pays d'origine, mais aussi les pays d'accueil aggravent la situation de ces migrantes d'Europe de l'Est par leurs régimes migratoires restrictifs.

\section{Le cadre politique: les régimes migratoires}

L'économie mondiale du care est ancrée dans des régimes migratoires et de l'emploi (supra)nationaux, qui entraînent des positionnements différents des travailleuses du care sur le marché du travail de leur pays d'accueil. En ce qui concerne la nationalité, il s'agit de différencier entre la mobilité des citoyennes et celle des non-citoyennes de l'UE. Dans le cas des Polonaises employées du care, la liberté de circulation est garantie par la législation de l'UE; tandis que les migrantes des pays voisins comme l'Ukraine, la Biélorussie, la Moldavie et la Georgie sont exclues de cette liberté de mouvement. Ce phénomène a des répercussions importantes sur leurs conditions de vie et de travail. Depuis 2011, de «nouvelles Européennes» (depuis 2014 des Bulgares et des Roumaines aussi) bénéficient de la liberté de circulation, qui vaut pour tous-toutes les citoyen-ne-s de l'UE ayant une activité professionnelle en tant qu'employé-e ou en tant que travailleur-se indépendant-e. Auparavant, ils-elles pouvaient seulement recourir au 'droit de libre circulation des services', et proposer leur force de travail sur le marché du travail allemand en tant que prestataire de services indépendant ou en tant qu'employé-e d'une entreprise dont le siège se trouvait dans leur pays d'origine.

Malgré la libéralisation du droit de séjour et de travail, la plupart des tra- 
vailleuses migrantes du care venues d'Europe de l'Est demeurent dans des rapports de travail non déclarés (pour le cas de la Pologne voir Neuhaus et al. 2009, pour celui de l'Ukraine Solari 2010), ou passent d'un statut non déclaré à un statut déclaré et viceversa $^{7}$. Étant donné que l'emploi non déclaré dans le travail domestique et de soins reste confiné dans l'espace privé des ménages, il est difficilement contrôlable. Des chercheurs-chercheuses soulignent qu'une légalisation et une régularisation des rapports de travail seraient peu rentables, tant pour les employeurs-ses que - du moins à court terme - pour leurs employé-e-s (Geissler 2006). De nombreux pays, dont la France (Scrinzi 2010) et l'Autriche (Schmid 2009), ont tenté de régulariser le travail domestique (des migrant-e-s). L'Allemagne a imposé un programme de recrutement restrictif des employées domestiques d'Europe de l'Est qui exclut le regroupement familial, ce qui est d'ailleurs en contradiction avec la législation de l'UE en vigueur. Karakayali (2010) constate que les employées en situation régulière et les travailleuses du care indépendantes sont elles aussi exposées à des conditions de travail précaires, qui diffèrent peu des conditions de travail des employées non déclarées, en particulier quand elles vivent dans les foyers au sein desquels elles travaillent et où elles font un travail de care 24 heures sur 24 . À cela s'ajoute la criminalisation dudit 'travail faussement indépendant': les migrantes qui travaillent pour une seule personne - ce qui est la norme dans le travail de care de personnes âgées dépendantes - tombent sous le coup de la loi et sont dans certains cas expulsées (Dollinger 2008).

Dans les pays d'accueil, non seulement les familles qui ont recours aux services de care, mais aussi les budgets de l'État tirent profit de la flexibilité des travailleuses du care. Tant que les migrations se limitent à des engagements présentant un intérêt économique immédiat, la migration temporaire demeure particulièrement attractive, étant donné que les coûts du travail reproductif, les risques sociaux et la responsabilité de la production du capital humain sont assumés par les sociétés d'émigration. Hormis la "charge» de leurs membres dépendants, qui - dans la perspective des pays d'accueil - doivent rester dans leur pays d'origine, les migrantes sont les bienvenues en tant qu'employées flexibles; cependant leur intégration sociale et culturelle à plus long terme n'est pas envisagée. Ainsi, par exemple, les employé-e-s migrant-e-s ne sont pas enregistré-e-s dans les systèmes d'assurance sociale des pays d'accueil et sont exclu-e-s des allocations-chômage, des retraites et des obligations du Code du Travail ${ }^{8}$. En résumé, on peut constaterque bien qu'ils soient hautement dépendants de ces forces de travail, les États membres de l'UE poursuivent une politique migratoire restrictive face aux employées du care, qui passent pour peu qualifiées, et les maintiennent ainsi dans une situation précaire. Pour résumer, cela signifie que leurs droits sociaux ainsi que ceux des membres de leur famille ne sont suffisamment protégés ni dans leur pays d'origine, ni dans leur pays d'accueil.

\section{Conclusion: la conciliation entre emploi et famille dans une Europe divisée}

En même temps que la transformation systémique de l'Europe de l'Est sur le plan historique, un changement promu sur le plan politique s'est opéré dans l'Union Européenne: les femmes sont davantage incitées à aller et à rester sur le marché du travail. Le recul de la participation des mères au marché du travail, observé dans plusieurs pays membres, a été qualifié par des économistes $d$ ' " anéantissement des ressources en capital humain féminin » (OECD 2001, p. 129) et a été vivement critiqué.

Au début du Xxi ${ }^{\mathrm{e}}$ siècle, on assiste à un rapprochement des politiques auparavant extrêmement différentes concernant l'activité professionnelle de la femme. Des femmes de «l'ancienne» comme de la "nouvelle» Europe on attend qu'elles s'engagent sur le marché du travail, et qu'elles complètent ainsi le revenu de leur ménage. Mais tandis que dans de nombreux pays de l'Ouest et du Sud les femmes de classe moyenne peuvent s'offrir les aides indispensables à cela, les femmes migrantes d'Europe de l'Est continuent de remplir leur obligation d'activité salariée (qu'elles ont acquise par l'Histoire); mais elles ne peuvent cependant plus concilier cette activité avec leurs obligations familiales. Elles doivent tenter de remplir leurs tâches parentales à distance.

Ainsi, le rideau de fer continue d'avoir un impact sur l'(in)égalité sociale en Europe. Dans le cas de la Pologne, qui est simultanément un pays pourvoyeur et receveur de maind'œuvre, un autre élément de ce rideau $\mathrm{du}$ care devient particulièrement visible: le lien entre lieu géographique et classe sociale. Dans ce pays en effet, ce sont les membres de la couche moyenne aisée qui emploient des travailleuses du care venues d'Ukraine, mais elles n'émigrent pas elles-mêmes vers l'ouest en tant qu'employées du care. On constate donc que le concept de "chaîne globale du care » dans sa constitution polarisante (les migrations Sud-Nord) ne s'applique que de façon partielle à l'Europe, car d'une part on ne trouve pas en Europe de l'Est certains des aspects spécifiques du Sud (les États socialistes comptaient parmi les leaders mondiaux pour leur niveau d'éducation élevé), et d'autre part l'appauvrissement collectif de la population y est une conséquence de la transformation du régime et de l'intégration dans le système capitaliste du marché mondial.

En plus des disparités économiques persistantes entre l'Europe de l'Est et de l'Ouest/et du Sud, les inégalités économiques à l'intérieur et entre les États nationaux de l'Europe de l'Est augmentent (OECD 2011), et se manifestent notamment dans les migrations du care est-est d'Ukrainiennes vers des foyers polonais.

Nous présumons cependant que les différences est-est forment un phénomène passager, qui pourrait être résorbé dans le cas de l'Ukraine par une entrée dans l'UE. 
Il est à noter qu'aucune migration de care d'Européennes du sud vers le nord (par exemple vers l'Allemagne) ne s'est produite après 2008, en dépit de la crise économique. Cela indique qu'à elle seule la situation économique n'est nullement décisive dans la décision d'émigrer. C'est au contraire en ayant recours à l'Histoire de la division de l'Europe que l'on peut répondre à la question de savoir si et pourquoi des femmes ayant un niveau d'éducation élevé sont néanmoins prêtes à se soumettre à des conditions de travail extrêmement individualisantes et frisant l'exploitation.

De façon surprenante, aucun débat approfondi ne s'est développé nulle part sur la question de qui devrait prendre en charge les enfants et les personnes âgées quand le travail salarié des femmes devient une obligation civique; ni dans «l'ancienne» ni dans la «nouvelle» Europe.

Dans son ouvre La condition de l'homme moderne (1958, traduction française 1961), Hannah Arendt attribue à deux grands économistes théoriciens de la modernité, Adam Smith et Karl Marx, l'origine de la distinction entre travail improductif et travail productif, et la critique du travail domestique comme improductif. Tous deux, selon Arendt, méprisaient le 'personnel domestique de maison' et en rabaissaient les pénibles labeurs en le considérant comme parasitaire et «comme une sorte de perversion, comme si rien n'était digne du nom de travail à moins d'enrichir le monde» (Arendt 2002, p. 131, traduit de l'anglais par Georges Fradier). Ainsi, avant l'apparition de la modernité, le travail domestique aurait été assimilé à l'esclavage, et, avec la société bourgeoise, au travail féminin.

Comme nous l'avons montré, ce point de vue a été repris par les précurseur-se-s du socialisme; ils-elles avaient cependant une idée précise de la façon dont le travail domestique des femmes devait être remplacé, ou facilité, par des services publics. Pourtant ce sont des rapports de genre bien traditionnels qui se sont consolidés durant le socialisme d'État, et qui se sont avérés bien plus résistants au changement qu'on ne l'aurait attendu.
Ce que les utopistes et les visionnaires sociaux-ales n'ont cependant jamais remis en cause, c'est la dévalorisation initiale du travail domestique et $\mathrm{du}$ travail de soins (care). Une Europe unie ne peut et ne devrait pas uniquement se pencher sur les asymétries économiques et sociales contemporaines, mais aussi sur une réévaluation de ce type de tâches, afin qu'elles soient enfin considérées comme un travail qui est en lui-même productif, nécessaire et vital pour l'existence humaine.

\section{Traduction Elise Pape}

\section{Bibliographie}

Arendt, Hannah (1958): The Human Condition, Cambridge. Version allemande (1958) Vita Activa oder Vom tätigen Leben, MünchenZürich. Traduction française de G. Fradier ([1961] 2002) Condition de l'homme moderne, Paris.

Backes, Gertrud M./ Amrhein, Ludwig/ Wolfinger, Martina (2008): Gender in der Pflege. Herausforderung für die Politik, FriedrichEbert-Stiftung, Bonn.

Böllert, Karin/Oelkers, Nina (Hrsg.) (2010): Frauenpolitik in Familienhand? Neue Verhältnisse in Konkurrenz, Autonomie oder Kooperation, Wiesbaden.

BMFSFJ - Bundesministerium für Familie, Senioren, Frauen und Jugend (2010): Familienreport 2010. Leistungen, Wirkungen, Trends, abrufbar unter: http://www.bmfsfj.de/RedaktionBMFSFJ/Broschuerenstelle/Pdf-Anlagen/ familienreport -2010 , property $=$ pdf,bereich $=b$ mfsf),sprache $=$ de,rwb=true.pdf $($ letzter Zugriff 20.12.2012)

Daly, Mary (2011): What adult worker model? À critical look at recent social policy reform in Europe from a gender and family perspective, in: Journal of European Social Policy 18, p. 1-23.

Dollinger, Franz-Wilhelm (2008): Von der Schwarzarbeit zur legalen pflegerischen Dienstleistung. Wie wir den Status der osteuropäischen Pflegerinnen legalisieren können. Gesundheitspolitisches Seminar 26.04.2008, Erbacher Hof, Mainz. Im Auftrag der KonradAdenauer-Stiftung e.V., abrufbar unter: http:// www.kas.de/wf/doc/kas_13717-544-1-30.pdf (letzter Zugriff 13.01.2013).

Dziennik.pl vom 27.3.2008: „W Polsce rosnie pokolenie Eurosiero“/„In Polen wächst die Generation der Eurowaisen auf", abrufbar unter: http://wiadomosci.dziennik.pl/ wydarzenia/artykuly/73098,w-polsce-rosniepokolenie-eurosierot.html (letzter Zugriff am 23.10.2013)
Engels, Friedrich ([1884] 1974): Der Ursprung der Familie, des Privateigentums und des Staates, Berlin.

Esping-Andersen, Gøsta (1990): The three worlds of welfare capitalism, Cambridge.

Eurofund - European Foundation for the Improvement of Living and Working Conditions (2006): Working time and work-life balance in European companies. Establishment Survey on Working Time 2004-2005, abrufbar unter: http://www.eurofound.europa.eu/ pubdocs/2006/27/en/1/ef0627en.pdf (letzter Zugriff 17.01.2013)

Geissler, Birgit (2006): Haushalts-Dienstleistungen als informelle Erwerbsarbeit. Neue Ungleichheit oder Ausdifferenzierung des Arbeitsmarktes?, in: Arbeit. Zeitschrift für Arbeitsforschung 15, p. 194-205.

Giza-Poleszczuk, Anna (2007): Rodzina i system społeczny, in Mirosława Marody (ed.): Wymiary życia społecznego. Polska na przełomie XX i XXI wieku, Warszawa.

Heimeshoff, Lisa Maria (2011): Europe. In: Helen Schwenken/Lisa Maria Heimeshoff (eds.): Domestic Workers Count. Global Data on an often Invisible Sector. Kassel: Kassel University Press, p. 45-56. http://www.uni-kassel.de/ upress/online/frei/978-3-86219-050-8.volltext. frei.pdf (letzter Zugriff am 16.5.2014)

Hess, Sabine (2005) : Globalisierte Hausarbeit. AuPair als Migrationsstrategie von Frauen aus Osteuropa, Frankfurt/Main.

ILO - International Labour Organization (2013): Domestic workers across the world. Global and regional statistics and the extent of legal protection, abrufbar unter: http://www.ilo. org/wcmsp5/groups/public/---dgreports/--dcomm/---publ/documents/publication/ wcms_173363.pdf (letzter Zugriff 17.01.2013).

Isaksen, Lise Widding (2010): Global Care Work. Gender and Migration in Nordic Societies, Lund/Sweden.

ISTAT - Istituto Nazionale di Statistica (2011): Demografia in Chifre, abrufbar unter: http:// demo.istat.it (letzter Zugriff 17.01.2013).

IWK - Institut der deutschen Wirtschaft Köln (2009): Arbeitsplatz Privathaushalt. Ein Weg aus der Schwarzarbeit. Pressekonferenz, 24. Februar 2009, Berlin, abrufbar unter: http://tinyurl.com/ckfn4hr (letzter Zugriff 24.05.2012).

Karakayali, Juliane (2010): Transnational Haushalten. Biographische Interviews mit Migrantinnen aus Osteuropa, Wiesbaden.

Kastner, Bernd (2008): Gesetzlose Hilfeleistung, in: Südddeutsche Zeitung 25 Januar 2008, p. 41.

Klaveren, Maarten van u.a. (2010): An overview of women's work and employment in Ukraine. Decisions for Life MDG3 Project Country Report $\mathrm{n}^{\circ} 8$, abrufbar unter: http://www. uva-aias.net/uploaded_files/publications/ WP94-Klaveren,Tijdens,Hughie-Williams,Ramos-Ukraine.pdf (letzter Zugriff 11.11. 2010). 
Lewis, Jane (2004): Auf dem Weg zur „ZweiErwerbstätigen"-Familie, in Sigrid Leitner/Ilona Ostner/Margit Schratzenstaller (eds.), Wohlfahrtsstaat und Geschlechterverhältnis im Umbruch. Was kommt nach dem Ernährermodell? Wiesbaden, p. 62-84.

Lutz, Helma (2008): Migration and Domestic Work. À European Perspective on a Global Theme, Avebury.

Lutz, Helma (à paraître) Behind Europe's Care Curtain. Migration and the Global Market of Care.

Lutz, Helma/Palenga-Möllenbeck, Ewa (2010): Care work migration in Germany: Semi-compliance and complicity, in: Special Issue Social Policy and Society 9, p. 419-430.

Lutz, Helma/Palenga-Möllenbeck, Ewa (2011): Das Care-Chain-Konzept auf dem Prüfstand. Eine Fallstudie der transnationalen CareArrangements polnischer und ukrainischer Migrantinnen, in Gender. Zeitschrift für Geschlecht, Kultur und Gesellschaft 3, p. 9-27.

Lutz, Helma/ Palenga-Möllenbeck, Ewa (2014): Care-Migrantinnen im geteilten Europa - Verbindungen und Widersprüche in einem transnationalen Raum. Soziale Welt 20, p. 217-231.

Menschik, Jutta/Leopold, Evelyn (1974): Gretchens rote Schwestern. Frauen in der DDR, Frankfurt am Main

Mikuła, Joanna (2008): Eurosieroctwo 2008. Warszawa: Studium Prawa Europejskiego: Instytut Europejski, abrufbar unter: http:// www.fundacja-pe.nazwa.pl/joomla/pliki/eurosieroctwo.pdf (letzter Zugriff 10.01.2013).

Minijob-Zentrale (2011): Trendreport. Alltag statt Luxus: Wie sich die Rolle von Haushaltshilfen verändert, Knappschaft-Bahn-See, abrufbar unter: http://www.minijob-zentrale. de/DE/Service/03_service_rechte_navigation/ DownloadCenter/8_trendreport/2011.pdf? blob=publicationFile\&v=2 (letzter Zugriff 10.01.2013)

Neuhaus, Andrea/Isfort, Michael/ Weidner, Frank (2009): Situation und Bedarfe von Familien mit mittel- und osteuropäischen Haushaltshilfen, Deutsches Institut für angewandte Pflegeforschung e.V., Köln, abrufbar unter: http://www.dip.de/fileadmin/data/pdf/ material/bericht_haushaltshilfen.pdf (letzter Zugriff 10.04.2010)

OECD (2001): Employment outlook. Kapitel 4: Balancing work and Family Life: Helping parents into paid employment, abrufbar unter: http://www.oecd.org/social/familiesandchildren/2079435.pdf (letzter Zugriff 13.01.2013).

OECD (2011). An Overview of Growing Income Inequalities in OECD Countries: Main Findings. Retrieved from http://www.oecd.org/ dataoecd/40/12/49499779.pdf (letzter Zugriff 24.07.2013)

Plomien, Ania (2009): Welfare state, gender, and reconciliation of work and family in Poland. Policy developments and practice in a new EU member, in Social Policy \& Administration 43 p. 136-151.

Rerrich, Maria S. (2012): Migration macht Schule. Herausforderungen für Care in einer rumänischen Gemeinde, in Mittelweg 36 5, p. 73-93.

Saraceno, Chiara/Keck, Wolfgang (2011): Towards an Integrated Approach for the Analysis of Gender Equity in Policies Supporting Paid Work and Care Responsibilities, in Demographic Research 25, p. 371-406.

Saxonberg, Steven/Szelewa, Dorota (2007): The Continuing Legacy of the Communist Legacy? The development of family policies in Poland and the Czech Republic, in Social Politics 14, p. 351-379.

Schmid, Tom (2009): Hausbetreuung - die Legalisierungs-Policy in Österreich, in Christa Larsen / Angela Joost / Sabine Heid (Eds.), Illegale Beschäftigung in Europa. Die Situation in Privathaushalten älterer Personen, München, p. 53-82.

Scrinzi, Francesca (2010): Masculinities and the International Division of Care. Migrant Male Domestic Workers in Italy and France, in Men and Masculinities 13, p. 44-64.

Scrinzi, Francesca (2013): Genre, Migrations et Emplois Domestiques en France et en Italie. Construction de la Non Qualification et de l'Altérité Ethnique, Paris.

Sikorska, Małgorzata (2009): Nowa matka, nowy ojciec, nowe dziecko. O nowym układzie sił w Polskich rodzinach. Warszawa.

Solari, Cinzia (2010): Resource Drain vs. Constitutive Circularity. Comparing the Gendered Effects of Post-Soviet Migration Patterns in Ukraine, in Anthropology of East Europe Review 28, p. 215-238.

Statistisches Bundesamt (2013): Pflegestatistik 2011. Wiesbaden.

Szelewa, Dorota/Polakowski, Michal P. (2008): Who cares? Changing Patterns of Childcare in Central and Eastern Europe, in Journal of European Social Policy 18, p. 115-131.

Walczak, Bartłomiej (2008): Społeczne, edukacyjne i wychowawcze konsekwencje migracji rodziców i opiekunów prawnych uczniów szkołach podstawowych, gimnazjalnych ponadgimnazjalnych. Warszawa, abrufbar unter: http://www.brpd.gov.pl/eurosieroctwo/raport_eurmigracje.pdf (letzter Zugriff 17.11.2008)

Winker, Gabriele (2011): Soziale Reproduktion in der Krise - Care Revolution als Perspektive, in Das Argument 53, p. 333-344.

Wippermann, Carsten (2011): Haushaltsnahe Dienstleistungen. Bedarfe und Motive beim beruflichen Wiedereinstieg, Bundesministerium für Familie, Senioren, Frauen und Jugend, Berlin, abrufbar unter: http://www.bmfsfi.de/ RedaktionBMFSFJ/Broschuerenstelle/PdfAnlagen/haushaltsnahe-dienstleistung,prope rty $=$ pdf,bereich $=$ bmfsfi,sprache $=$ de, $r w b=$ true . pdf (letzter Zugriff 10.01.2013).

\section{Notes}

* Cet article est une version raccourcie d'un texte portant le même titre, qui est paru dans la revue allemande Soziale Welt en 2014. Il a été traduit de l'allemand vers le français par Elise Pape. Toutes les citations d'auteur-e-s qui figurent dans le texte ont été traduites par la traductrice.

1. La France et les pays scandinaves constituent cependant des exceptions importantes à cet égard: l'État y fixe en partie d'autres priorités, par exemple concernant les services de la petite enfance ou une scolarisation visant une éducation à la citoyenneté (dans le cas de la France) ou la pleine exploitation des ressources de l'activité professionnelle des femmes (dans le cas le cas de la Scandinavie).

2. Cet article repose sur un matériel recueilli dans le cadre du projet de recherche Landscapes of Care Drain. Care Provision and Care Chains from the Ukraine to Poland and from Poland to Germany [Paysages du Care Drain. Les services du care et les chaînes de care de l'Ukraine vers la Pologne et de la Pologne vers l'Allemagne] effectué entre 2007 et 2010. Ce projet a été financé par la Deutsche Forschungsgemeinschaft [l'équivalent de l'Agence Nationale de la Recherche pour l'Allemagne] dans le cadre du programme EUROCORES. Dans ce projet, le matériel suivant a été recueilli en Ukraine, en Pologne et en Allemagne: (1) Une analyse secondaire des régimes migratoires, des politiques de rapports de genre et des politiques du care; (2) Une analyse de discours d'articles de presse portant sur le care et les migrations; (3) 22 récits de vie avec des migrantes polonaises et ukrainiennes et 41 entretiens semi-directifs avec des membres de leurs familles (leurs époux, les grands-parents, les enfants et ami-e-s) restés en Pologne et en Ukraine.

3. En anglais: Adult Worker Model.

4. Avec l'exception des pays mentionnés cidessus.

5. Nous avons analysé trois quotidiens allemands: la Frankfurter Allgemeine Zeitung (FAZ), la Süddeutsche Zeitung (SZ) et la Bild-Zeitung.

6. Les études de cas présentées ici se limitent aux migrantes polonaises, elles concernent cependant de façon similaire les Ukrainiennes de notre échantillon.

7. Ceci entre autres pour économiser les impôts et les charges sociales élevés.

8. Ici la France et la Belgique font exception depuis qu'y a été institué dans les années 2000 le Chèque emploi service, grâce auquel les employeurs privés ont tout intérêt à déclarer à la Sécurité sociale leur salarié-e et à payer leurs charges sociales 
'patronales'. Les employé-e-s deviennent ainsi, quelle que soit leur nationalité, des assuré-e-s sociaux-ales ordinaires bénéficiant d'assurance maladie, de l'assurance chômage et de droits à une pension de retraite. Bien qu'apportant certaines améliorations, des travaux récents montrent cependant que la rémunération du travail de care et les possibilités d'avancement professionnel ou de sortie de ce secteur d'emploi restent faibles (Scrinzi 2013). 